\section{Effect of Notched Strings on Tennis Racket Spin Performance: Ultrahigh-Speed Video Analysis of Spin Rate, Contact Time, and Post-Impact Ball Velocity*}

\author{
Yoshihiko KAWAZOE**, Yukihiro TAKEDA** and Masamichi NAKAGAWA** \\ **Department of Mechanical Engineering, Saitama Institute of Technology, \\ 1690 Fusaiji, Fukaya-shi, Saitama 369-0293, Japan \\ E-mail: kawazoe@sit.ac.jp
}

\begin{abstract}
While some tennis racket strings have more grip than others do, this does not guarantee that they will impart more spin to a tennis ball. Experiments with hand-held rackets are required to determine the longstanding question of how players can discern that different strings behave differently when laboratory tests indicate that they should play the same. In a previous study, we clarified the top-spin mechanism of a tennis racket by using high-speed video analysis on a tennis court for the first time. Furthermore, we improved it by using lubricated notched nylon strings. These experiments revealed that the more the main strings stretch and bend laterally, the more spin is imparted to the ball. This is due to the restoring force being parallel to the string face when the main strings spring back and the ball is released from the strings. Notched strings reduce the spin rate, but this can be effectively counteracted by employing lubricants. Furthermore, we found that imparting more spin reduces shock vibrations on the wrist during impact. The present study revealed that a ball has a $40 \%$ lower spin rate when hit with a racket with notched strings than with one with unnotched strings in the case of nylon (it had to be determined whether new strings or lubricated used strings give more spin). The experiments also showed that $30 \%$ more spin is imparted to a ball when the string intersections are lubricated by oil than when notched used nylon strings are used. Furthermore, we found that used natural gut notched strings reduced the spin rate by $70 \%$ compared to when new natural gut unnotched strings are used. We also investigated different top-spin behaviors obtained when professional and amateur tennis players hit a ball.
\end{abstract}

Key words: Sport Engineering, Tennis Racket, Impact, Ball Spin, Strings, Notch, Ultrahigh-Speed Video Analysis, Shock Vibrations, Tennis Elbow

\section{Introduction}

The degrees of freedom in the manufacture and design of tennis rackets are increasing due to a greater diversity of composite materials. Rackets are now designed by carefully considering the physical and technical characteristics of players. A wide variety of rackets are now commercially available, including some that enhance the feel when hitting a ball by incorporating piezoelectric elements in the neck of the racket or pulleys in the grommets.

Progress is being made in constructing databases and developing systems to objectively evaluate racket performance (which has traditionally been assessed based on human senses),
Received 4 Aug., 2011 (No. T2-09-0741) Japanese Original : Trans. Jpn. Soc. Mech. Eng., Vol.76, No.770, C (2010), pp.2646-2655 (Received 17 Aug., 2009) [DOI: 10.1299/jsdd.6.213]

Copyright $\odot 2012$ by JSME 
by considering the action of a player when they swing the racket and the collision between the racket and the ball. Furthermore, much has been revealed regarding factors such as rebound performance, usability, ball flight, and the sensation when hitting a ball. ${ }^{(1)-(10)}$

Tennis involves controlling a ball within a court. To achieve this control, a suitable amount of spin needs to be imparted to the ball. Balls with well applied spin will bounce sharply on hitting the ground making them difficult to return.

Rackets have become easier to use due to having greater surface areas and being more lightweight so that it is no longer unusual for even general players to apply top spin. There is growing interest in the relationship between spin performance and the types of rackets and strings. However, despite having being investigated for a long time, it is still unclear how spin is affected by factors such as the type, material, gauge (strand diameter), and tension of strings, of which there are now several hundred commercially available.

While many players claim through experience that some strings "bite" the ball better than others ${ }^{(11)}$, recent, detailed experiments involving oblique collisions with a fixed racket head $^{(12),(13)}$ revealed no clear relationships between the spin performance of a racket and the string friction properties, types of strings, and the tension with which they are strung (i.e., their initial tension). Therefore, it is not clear how spin is imparted when a player strikes a ball. Although it is difficult to reproduce impacts, experiments in which players actually use rackets to hit balls are required to solve this problem (namely, that players can feel differences in strings even though such differences are not discernable in the laboratory), which has been discussed for some time.

Hence, in a previous study, ${ }^{(14)}$ we used ultrahigh-speed video analysis $(10,000 \mathrm{fps})$ of impacts using testing equipment on the court and collision simulations to clarify for the first time the puzzle regarding the spin performances of rackets.

Our findings include the following:

(1) Contrary to the conventional assumption that greater string friction results in more spin, when using low friction string, the main (vertical) and cross (horizontal in the string plain) strings slide across one another and the intersections slip. The spin rate is increased due to the restoring force when the main strings are stretched laterally and then spring back (Fig. 1).

(2) It is easier to apply spin to a ball when string intersections are lubricated.

(3) The contact time increases as the spin rate is increased.

(4) As the contact time increases, the shock transmitted to the racket and the hand decreases.

(5) "Spaghetti string" rackets ${ }^{(15)}$ (their use was banned some time ago because they could apply excessive spin by using plastic tubing to reduce friction) and rackets with lubricated string intersections have very similar spin mechanisms.

(6) It is easier to apply spin using low-friction strings such as polyester than nylon strings (known as "spin gut"), which have a high surface friction.

(7) Slippery, hard polyester strings have become the strings of choice for players ranging from the world's top-level professionals such as Nadal (who is famous for his powerful top spin) to amateurs.

In this experiment ${ }^{(14)}$, a racket was used for $3 \mathrm{~h}$ per day for a week, after which the notched nylon strings (Yonex Aeron Super 850) were lubricated with silicon oil (international patent acquired; it conforms with International Tennis Federation regulations). For top-spin shots, there was an average increase in the spin rate of $30 \%$, an average increase in contact time of $16 \%$, and an average reduction in post-impact ball velocity of $6 \%{ }^{(14)}$. 


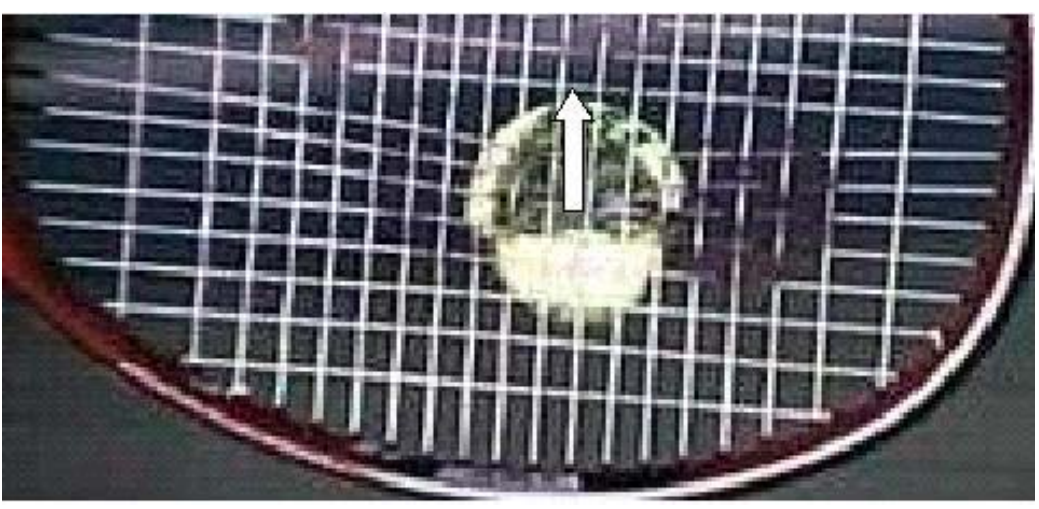

$1.7 \mathrm{~ms}$ after contact (T506_f159_L4)

Fig.1 Ball spin behavior and mechanism of spin rate increase by lubricating string intersections. Main strings stretch and move laterally before springing back when the ball is released from the strings.

In this study, we first compared the top-spin performances of notched nylon strings (Yonex Aeron Super 850) and brand-new unnotched strings. The results of our previous report, which suggested that the spin rate is considerably higher for rackets with notched strings and to which lubricant had been applied to the intersections ${ }^{(14)}$, were discussed by the International Tennis Federation (ITF) Technical Commission. We submitted samples of the lubricant and they were found not to violate the current rules, but a project is currently underway to establish rules relating to agents for producing spin from the strings and we have received notification that the rules may change in the future; this is the motivation for choosing this topic. The same series of experiments was performed as the previous study. ${ }^{(14)}$ They demonstrate that even when lubricant is applied to notched strings, their spin performance fails to reach that of brand-new unnotched strings.

This study does not aim to quantitatively investigate the relationship between the extent of notching or wear on strings and the spin rate. It aims to highlight three points: the presence of notches at the string intersections considerably reduces the spin performance; facilitating sliding of the main (vertical) and cross (horizontal in the string plain) strings at impact makes it easier to apply spin; and contrary to conventional wisdom (which differ from the descriptions of "spin gut" given in catalogs), using lower-friction string materials makes it easier to apply spin. This third point is demonstrated by the fact that almost all players (not just professionals who are skilled at applying top spin) have recently been using hard, wear-resistant polyester ${ }^{(14)}$ strings, which were barely used in the past. This implies that there is a real possibility of making ball control easier. Our main objective is to breathe new life into the conventional design concepts for strings and to carve out new frontiers.

In the case of gut (natural) strings, we compared the spin behaviors of notched gut and brand-new gut strings when a top-spin shot was performed by a professional tennis player. The reduction in the spin performance of notched gut was quantitatively investigated by ultrahigh-speed video analysis. A great variety of strings are commercially available ${ }^{(16)}$ and the performance of natural gut is of interest to many players. Of particular interest is the extent to which the spin performance is poorer in notched natural gut relative to that of brand-new gut.

In addition, we quantitatively compared and evaluated the spin behavior resulting from shots by professional and amateur players. A large aspect of tennis (and of sports in general) is subjective, as it is mastered by experience. As such, the performance of equipment varies depending on the player. Differences in the spin behaviors of balls hit with top spin by professionals and amateurs is of great interest. 


\section{Effect of notches on the spin performance of nylon strings}

The lubricant (for which our collaborators have acquired an international patent) ${ }^{(14)}$ considerably increases spin rates when applied to the intersections of notched strings. It does not currently violate ITF rules. However, a project is currently underway to establish rules relating to agents for producing spin and we have received notification that the rules may change in the future. Therefore, in this section, we verify that even when lubricant is applied to notched strings, their spin performance fails to reach that of brand-new unnotched strings. Some of these experiments are the same as those in the previous report $^{(14)}$ and the same testing equipment and measurement conditions are employed.

Figure 2 shows the spin behavior of a ball when hit with top spin by a racket strung with brand new nylon strings (Yonex Aeron Super 850) in a sequence of frames photographed from the side at 10,000 fps. Figure 2(a) shows the spin behavior during the contact time when the ball and strings are touching. It shows that spin is produced at this time. Figure 2(b) shows the rotation of the ball after it is released from the strings.

As in the previous report ${ }^{(14)}$, Fig. 3 shows like those of Fig. 2, which were taken at the moment of impact from directly beside the racket, and photographs from directly behind. It summarizes the results for ultrahigh-speed video analysis of top-spin shots for three cases: brand-new strings, strings used 3 hours a day for 1 week, and strings lubricated with silicon oil (international patent acquire; conforms with ITF rules) after 1 week of use. These are average values obtained from three trials and their standard errors. In top-spin shots, compared to brand-new strings, the racket used for 3 hours a day for about 1 week after stringing had an average reduction in spin rate of $40 \%$ and an average reduction in contact time of $8 \%$. However, when lubricant was applied, there was an average gain in spin rate of $30 \%$ and an average increase in contact time of $16 \%$. The average reduction in linear post-impact ball velocity was $6 \%{ }^{(14)}$ If lubricant is applied to notched strings, there is a large increase in spin rate, but it does not match the spin rate of brand new strings without notches.

Figure 4 shows the model used to determine the shock force transmitted to the hand at the moment the ball is struck by the player. Figure 5 shows the predicted location of shock vibration acceleration in the wrist. ${ }^{(2),(8),(10)}$

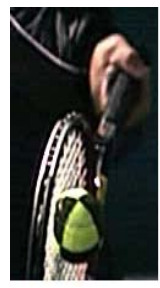

$0.0 \mathrm{~ms}$

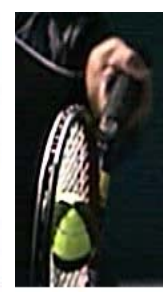

$1.0 \mathrm{~ms}$

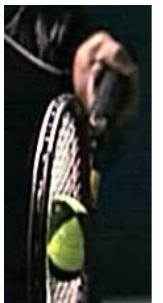

$2.0 \mathrm{~ms}$

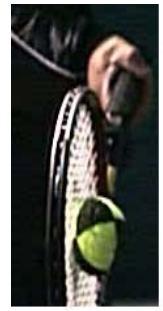

$3.0 \mathrm{~ms}$

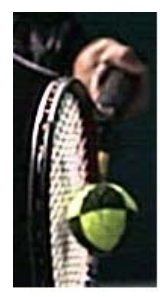

$3.8 \mathrm{~ms}$

(a) Contact time : Ball release $V_{B}=\mathbf{2 4 . 8} \mathrm{m} / \mathrm{s}$

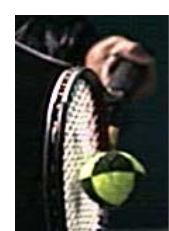

$1 \mathrm{~ms}$

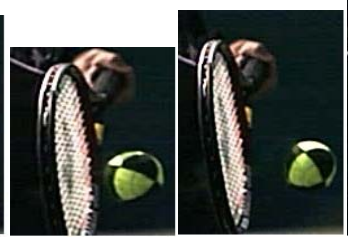

$2 \mathrm{~ms}$
$4 \mathrm{~ms}$

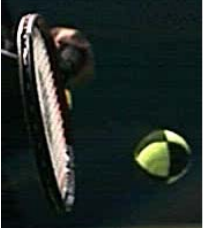

$6 \mathrm{~ms}$

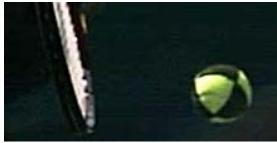

$8 \mathrm{~ms}$

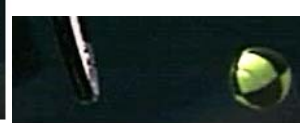

$10 \mathrm{~ms}$

(b) After release(f77-)

Fig. 2 Top-spin behavior with a newly strung tennis racket (Trial 003: f39 ) 


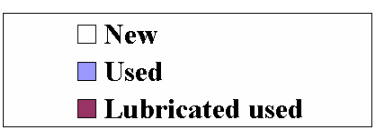

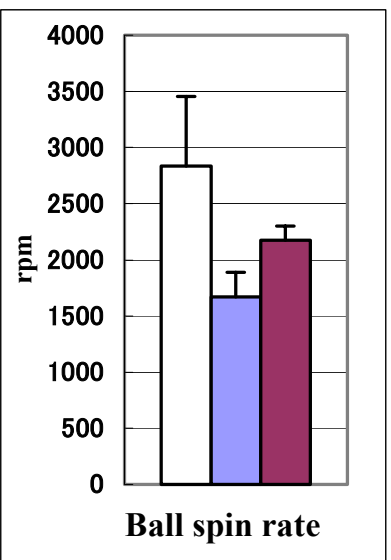

(a) Ball spin rate $\omega$

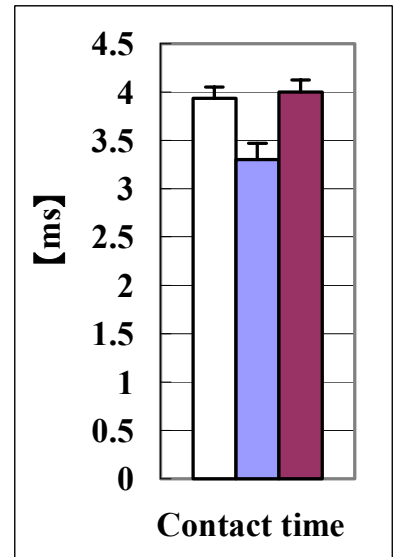

(b) Contact time $T_{C}$

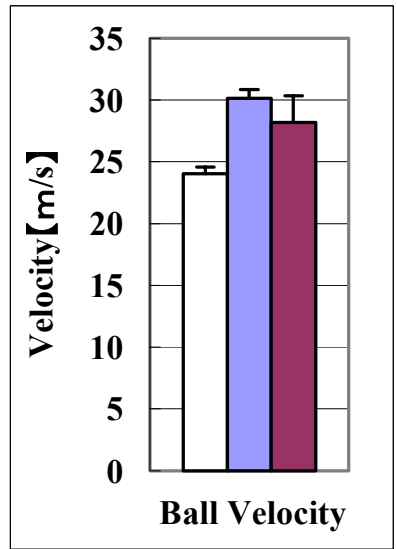

(c) Post-impact ball velocity $V_{B}$

Fig. 3 Spin performance vs. string conditions with mean and standard error.

The method used to calculate the shock vibration acceleration was omitted in our previous report $^{(14)}$, so we give an outline of it below.

At the moment of impact, gravity and muscle strength are assumed to be low compared to the collision force, and the arm joints are treated as a pin joints. The distance between the handle grip and the wrist is ignored and the force acting on the shoulder $S_{3}$ is assumed to have no effect on the velocity of the shoulder. Taking the collision force acting on the ball and strings to be $S_{0}$ and the forces acting on the player's wrist (P1), elbow (P2), and shoulder (P3) to be $S_{1}, S_{2}$, and $S_{3}$ respectively, the shock force components for the wrist, elbow, and shoulder joints can be found, as can the shock acceleration component. By performing experimental modal analysis of a racket supported by a hand and by identifying the attenuation from the acceleration waveform measured in hitting experiments, we can determine the vibration acceleration component at the position of the grip of the racket when it collides with a ball at an arbitrary point on the racket surface. In a forehand groundstroke, the acceleration waveform for the wrist joint measured when hitting with the racket coincides well with the predicted waveform, considering the shock component and the four vibration mode components; namely, the two-node bending, three-node bending, and two-node torsion modes of the frame vibration, and the first vibration mode of the string surface. The shock vibration acceleration waveform $A_{\text {grip }}$ is expressed (see below) by the sum of the shock acceleration component (the first term) and the vibration acceleration component (the second term): ${ }^{(2),(8),(10)}$

$$
A_{\text {grip }}=A_{n v} \sin \left(\frac{\pi t}{T c}\right)+\sum_{i=1}^{4} A_{v i} e^{-\sigma_{i} t} \sin 2 \pi f_{i} t
$$

where

$$
\begin{gathered}
A_{n v}(t)=S_{0}(t)\left[1 /\left(M_{R}+M_{H}\right)-\left(a / I_{G}\right) X\right], \\
A_{v i}(t)=-\left(2 \pi f_{i}\right)^{2} r_{i} S_{0}\left(2 \pi f_{i}\right), \\
\sigma=2 \pi f_{i} \zeta_{i} .
\end{gathered}
$$

$M_{R}$ is the mass of racket, $M_{H}$ is the reduced mass at the grip location in the arm system, $X$ is the distance between the center of gravity of the racket-arm system and the grip location, $a$ is the distance between the center of gravity of the racket-arm system and the collision point 
on the racket surface, $I_{G}$ is the moment of inertia around the center of gravity of the racket-arm system, $r_{i}$ is the residue between the grip position and the strike location on the surface of the racket. $S_{1 \max }$ is the largest shock force transmitted to the hand, which corresponds with $\mathrm{S}_{0 \text { max }}$, the largest collision force. $A_{v i}(t)$ is the $i$ th vibration mode component of the initial vibration acceleration amplitude at a position $70[\mathrm{~mm}]$ from the grip end. ${ }^{(8)}$ Average data taken from several scientific literatures with the weight and the height of a player were $60 \mathrm{kgw}$ and $170 \mathrm{~cm}$, respectively. The mass $M_{H}$ concentrated in the hand was estimated as $M_{H}=0.97 \mathrm{~kg}$, nearly $1.0 \mathrm{~kg}{ }^{(17)}$. The location of the center of gravity of the racket-arm system is about $130 \mathrm{~mm}$ from the end of racket handle ${ }^{(17)}$.
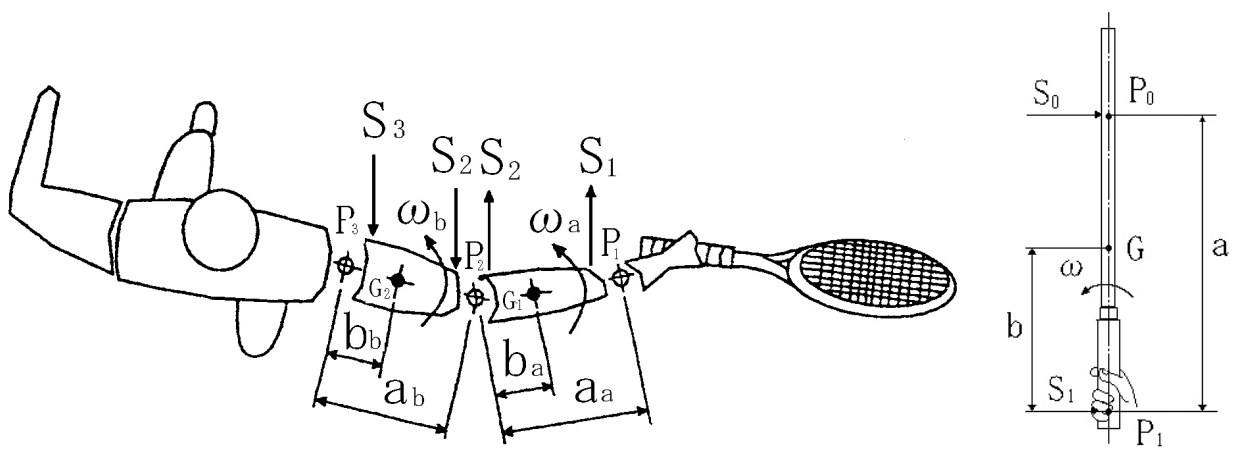

Fig. 4 Model used to predict the shock force transmitted to the arm joint from a racket.

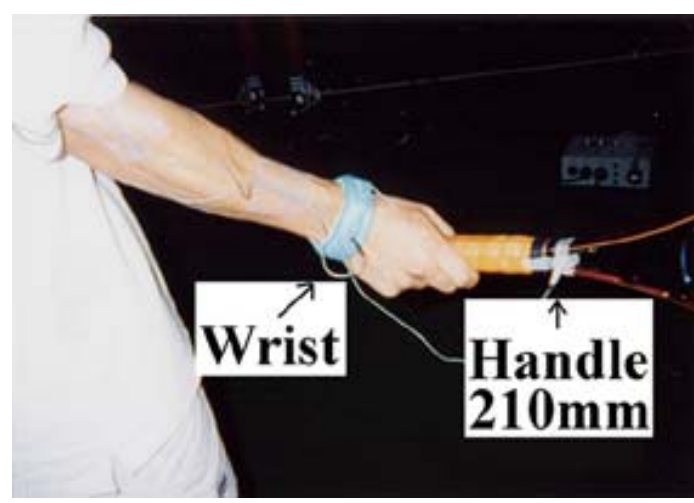

Fig.5 Locations of accelerometers on wrist for forehand ground stroke and the racket handle.

Figure 6 shows screen shots of the display showing the shock vibration waveforms for the wrist predicted by a system for predicting and evaluating tennis racket. ${ }^{(17),(18)}$ It represents the time $0.1 \mathrm{~s}$ after impact; the collision point with the ball is near the tip of the racket face ( $95 \mathrm{~mm}$ from the edge). The symbol $\mathrm{G}$ represents the acceleration due to gravity and it also represents the peak-to-peak (i.e., maximum and minimum) accelerations. Figure 6(a) shows the case with no spin at a collision velocity of $30 \mathrm{~m} / \mathrm{s}$. The shock vibration is substantial and there is considerable high-frequency vibration of the string. Figures $6(\mathrm{~b})$ and (c) show the cases when top spin is applied with notched strings and strings to which lubricant had been applied, respectively.

The vertical component impulses in Figs. 6(b) and (c) are respectively 0.63 and 0.49 times that in Fig. 6(a). Figure 6(d) shows the case for brand-new strings; the contact time is a little shorter than that of lubricated strings, but the shock vibration is approximately the same degree.

As the main strings slide laterally, the restoring force parallel to the string face gives rise to a higher spin rate, the contact time increases, and the vertical component of the collision force decreases. The flexural deformations of the ball and the strings thus decrease; video images (not shown) indicate that the entire string surface flexes slightly with only the region 
in contact with the ball sinking in. The racket frame vibration also decreases. This is thought to correspond with the subjective feel when hitting the ball with brand-new strings, which, according to testers, gives "increased sensation of biting the ball", "an increased sense of hold", and "a gentler feel when hitting the ball".

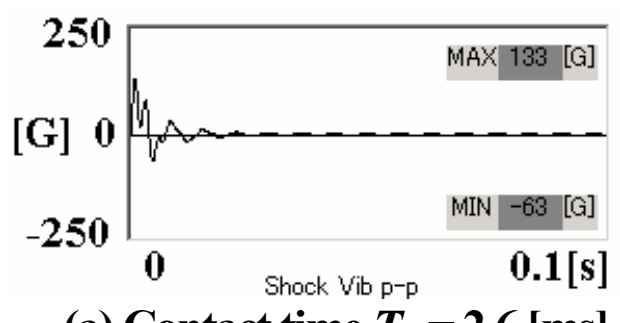

(a) Contact time $T_{C}=2.6[\mathrm{~ms}]$

(Flat impact)

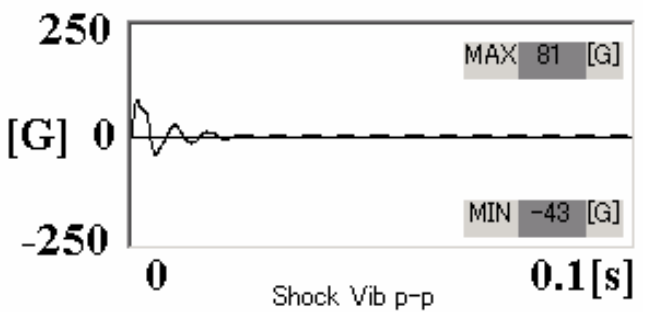

(b) Contact time $T_{C}=3.4[\mathrm{~ms}]$

(Top spin with used strings)

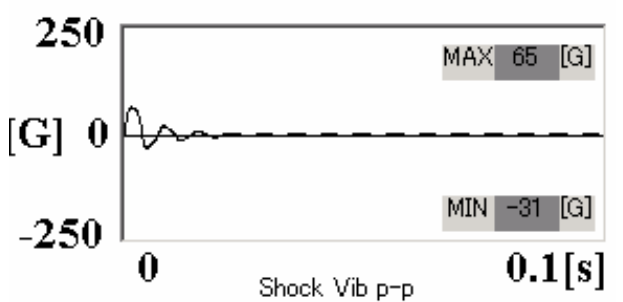

(c) Contact time $T_{C}=4.1[\mathrm{~ms}]$

(Top Spin with lubricated used strings)

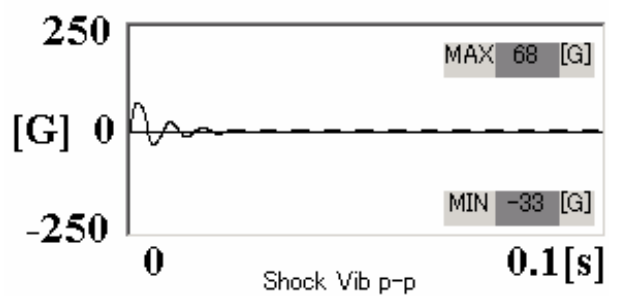

(d) Contact time $T \mathrm{c}=3.9 \mathrm{~ms}$ (Top Spin with new strings)

Fig.6 Calculated effect of contact time on the wrist shock vibrations (impact velocity: 30 $\mathrm{m} / \mathrm{s}$, hitting location: $95 \mathrm{~mm}$ from the tip of racket). The horizontal axis shows the time from the impact to $0.1 \mathrm{~s}$.

\section{Effect of notches on the spin performance of gut (natural strings)}

A great variety of strings are commercially available. Of these, natural gut is expensive but of interest to many players. An intriguing question is how much the spin performance of notched natural gut decreases relative to that of brand new gut. 
In this section, we investigate the spin behavior of natural gut (natural strings) for a ball hit with top spin by a professional tennis player and quantitatively study the reduction in spin performance for notched gut by ultrahigh-speed video analysis.

As stated in Section 1, it is extremely difficult to record clear, ultrahigh-definition images of impacts using actual players. Hence, highly reproducible professional ball release and spin shot hitting technologies are necessary, which can ensure impacts within the frame of a fixed ultrahigh-speed camera. For example, with today's commercially available ball machines, it is very difficult to feed the ball to a fixed location with a high reproducibility and the ball location will vary considerably. For ball feeding, we requested top-class players from all over Japan. The photography was the result of collaboration with NHK (Japan Broadcasting Corporation) and the Japan Tennis Association to overcome the difficult conditions.

In the experimental data presented below, we observed the spin behavior during shots played by a professional tennis player by ultrahigh-speed video analysis in the process of producing a television program.

Figure 7(a) shows the setup of cameras used for filming the spin behavior from the side and Fig. 7(b) shows that for photographing the spin trajectory from directly in front, and the transparent reflector equipment used to bounce the ball back. The cameras employed were the Memrecam fx 6000 high-speed color camera by NAC Image Technology Inc. (top speed: $210,000 \mathrm{fps}$ ) in the experiment in Section $2^{(14)}$, as well as the Ultra Cam HS-106E (1,250,000 fps, ultrahigh shutter speed: $100 \mathrm{~ns})$, ultrahigh-speed color camera. The HS-106E uses a CCD image sensor developed jointly by NHK's Science \& Technology Research Laboratories and Prof. Takeharu Eto of Kinki University, realized commercially

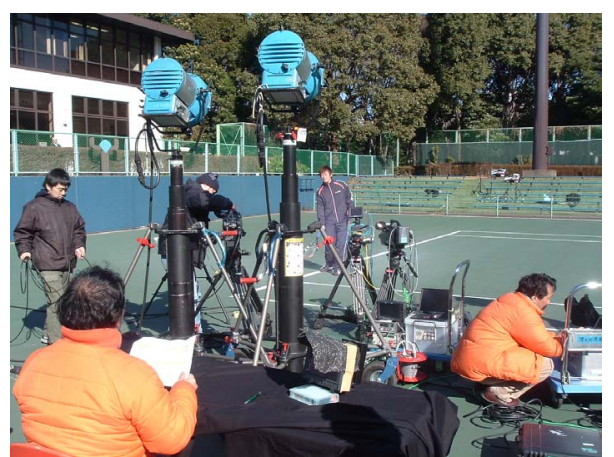

(a)

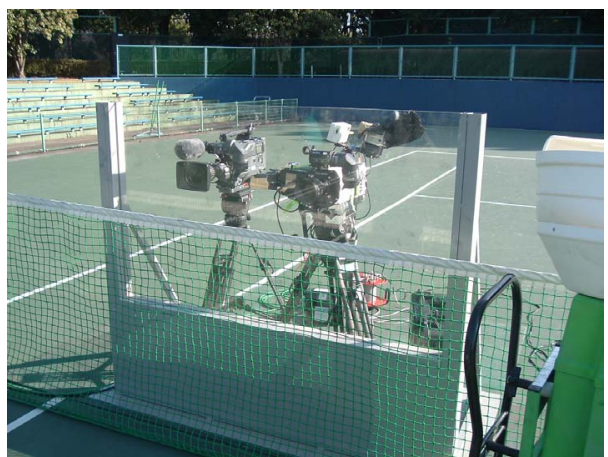

(b)

Fig.7 View of the experimental set up.

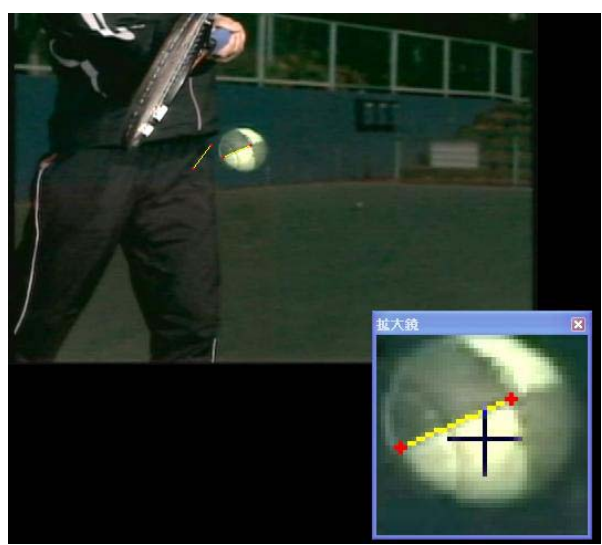

(a) Analysis of spin rate

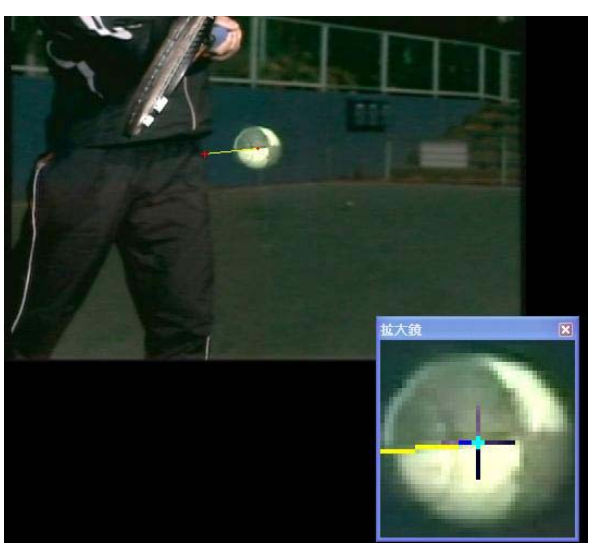

(b) Analysis of ball velocity

Fig. 8 View of impact and spin behavior analysis. 
by Hitachi Kokusai Electric Inc., and developed into a system and released by NAC Image Technology Inc. we used the remote control utility fxLink as well as in the experiment in Section 2.

Figure 8 shows example images that reveal the top-spin behavior of a ball that has been hit by a player. As in the experiment in Section 2, two-dimensional image analysis was mainly performed by the motion analysis software NAC's MOVIAS Pro-LAA. Although it is difficult to see in these photographs, the spin (rotation) rate can be determined from the angle of rotation of the ball with time (see Fig. 8(a)), and the linear velocity can be determined from the linear distance travel with time (see Fig. 8(b)).

Figure 9 shows a single frame of the footage taken from directly behind, which was primarily to analyze the contact time between the ball and the strings (in Section 3, this is natural gut; i.e., cattle intestine), from when they touch until they part, and the movement. It shows that the main strings slide laterally. In the present experiment, the photography from directly to the side and directly behind were not performed simultaneously; rather, they were performed separately. In Section 2, the contact area was analyzed as well as the contact time between the ball and strings. However, the objective of the present study is not to take detailed measurements of numerical values, but to focus on breathing new life into the design concept for strings and to forge new frontiers. Consequently, calculations of figures relating to contact area and the amount of string slippage are omitted.

Figure 10 shows a micrograph of the used gut employed in the experiment, which had previously been played with. Since this is a frame taken from a moving image, the contrast is poor, but the notches can clearly be seen.

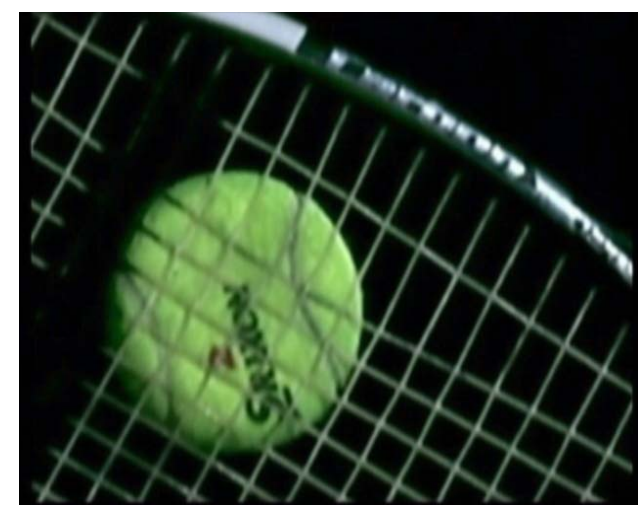

Fig.9 Ball spin behavior and the mechanism for increasing the spin rate in which strings stretch, slide laterally, and spring back the main strings at the string intersections when a ball is released from the string bed for the case of new gut.

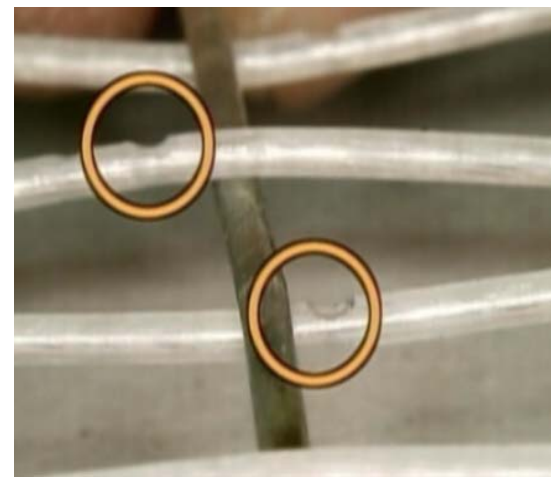

Fig.10 Used gut with notches at the intersections. 
Figure 11 gives an overview of the swing trajectory immediately before and after impact in the case of a top-spin shot performed by a leading professional Japanese tennis player. Figure 12 shows the top-spin behavior during the period in which the ball and gut are in contact. Figure 13 shows the spin behavior after the ball has left the strings. The ball has been marked to facilitate recognition of the rotation.

The frame pictures in Figs. 11 to 13 were analyzed to calculate the linear velocity and the angular velocity.

Figure 14 shows the impact behavior (from the start until the end of contact) photographed from behind a newly strung racket for a shot made by a professional tennis player. The application of rotation to the ball can be observed, as the main strings of the racket slide laterally, bite into the ball, and spring back.

Figure 15 is the impact behavior (from the start until the end of contact) when a top spin shot is performed by a professional tennis player using notched gut strings that had been used previously. For the racket with notched gut, there is slight lateral slippage of the main strings.

Figure 16 shows the spin rate and post-impact ball velocity (calculated from the frames shown in Figs. 11 to 13) and the contact time between the ball and the gut (calculated from Figs. 14 and 15). It compares the results of top-spin behavior analysis of shots performed by a professional using notched gut strings that had previously been used and brand-new gut strings that do not have any notches. Deep notches are formed at the intersections on the used strings that had previously been used and when a ball is hit with a top-spin technique, there is little restoring force caused by the lateral slippage and return of the main strings. This considerably reduces the spin rate $(70 \%$ reduction) and shortens the contact time (13\% reduction); these results support the top-spin mechanism described in our previous study. ${ }^{(14)}$

The top spin applied by a professional tennis player using brand new gut strings is approximately $50 \mathrm{rps}$. As we will report in a separate paper, this leads to post-bounce behavior that makes it difficult for the opponent to hit the ball. The post-impact velocity is faster in notched gut ( $50 \%$ increased) due to its substantially reduced spin rate. According to the video footage, the gut strings that had previously been used resulted in a spin rate equivalent to $30 \%$ that of the brand-new gut strings. Compared to the high bounce of the ball from the brand-new gut strings, which was far in excess of body height, the ball lacked vigor after bouncing.

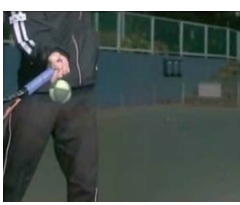

$0.0 \mathrm{~ms}$

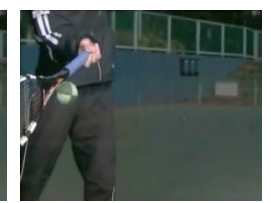

$7.5 \mathrm{~ms}$

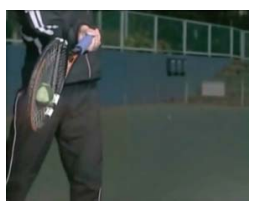

$15.0 \mathrm{~ms}$ (impact)

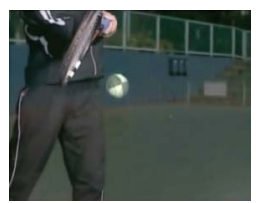

$22.5 \mathrm{~ms}$

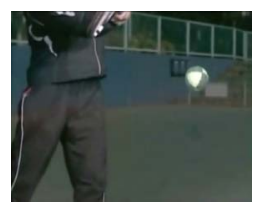

$30 \mathrm{~ms}$

Fig.11 Top-spin swing applied by a professional tennis player.

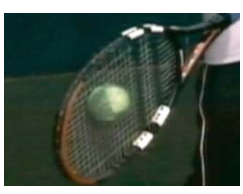

$0.0 \mathrm{~ms}$

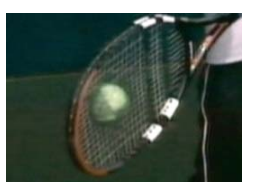

$1.0 \mathrm{~ms}$

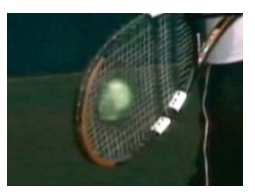

$2.0 \mathrm{~ms}$

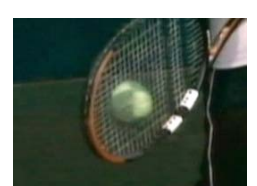

$3.0 \mathrm{~ms}$

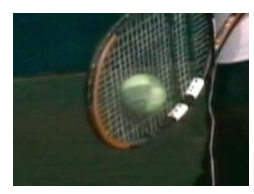

$4.0 \mathrm{~ms}$

release

contact

Fig.12 Images obtained during impact of a top-spin shot by a professional tennis player. 


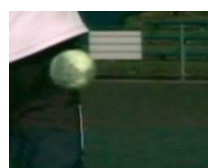

$0.0 \mathrm{~ms}$

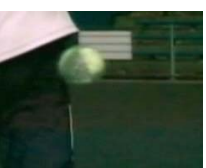

$1.0 \mathrm{~ms}$

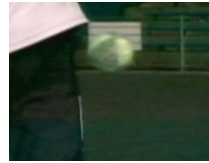

$2.0 \mathrm{~ms}$

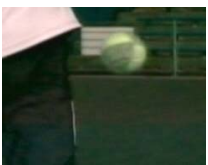

$3.0 \mathrm{~ms}$

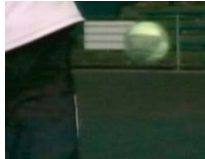

$4.0 \mathrm{~ms}$

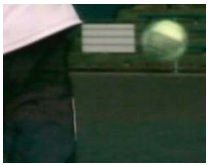

$5.0 \mathrm{~ms}$

after release

Fig.13 Side view of ball top-spin behavior by a professional tennis player using strings made from new gut (about $50 \mathrm{rps}$ ).

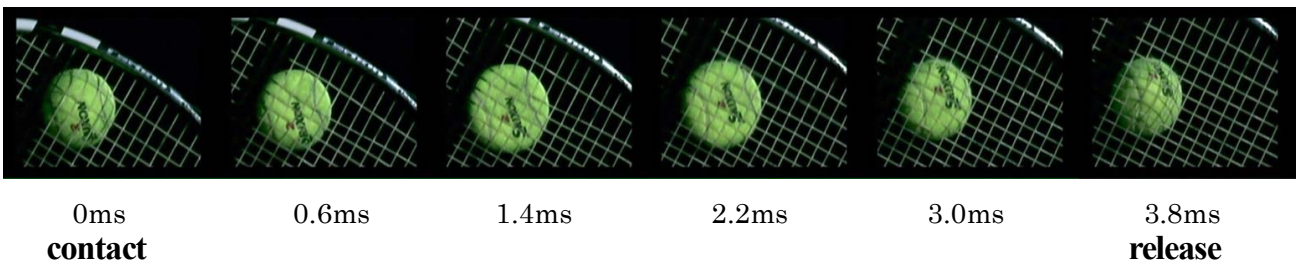

Fig.14 Top-spin behavior and spin rate increase mechanism in which the main strings stretch, slide laterally, and spring back at the string intersections for the case of new gut strings.

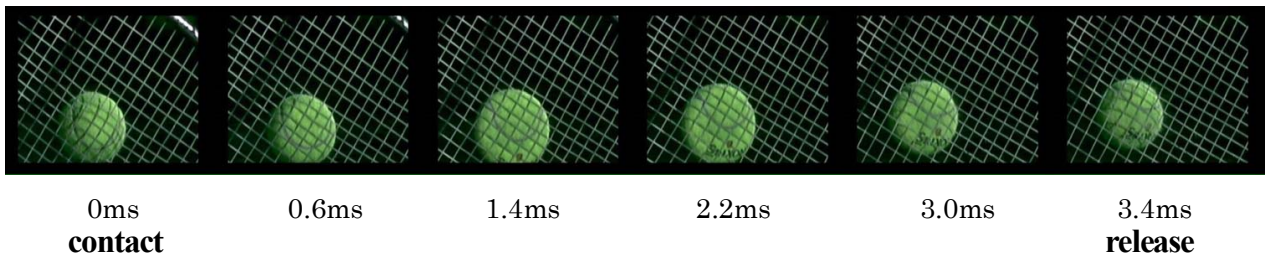

Fig.15 Top-spin behavior for used gut strings.

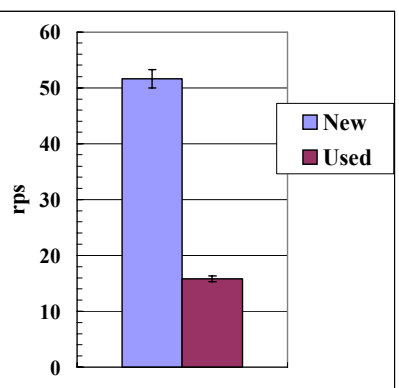

(a) Ball spin rate $\omega$

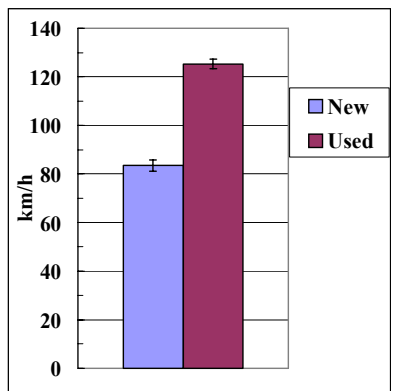

(b) Post-impact ball velocity $V_{B}$

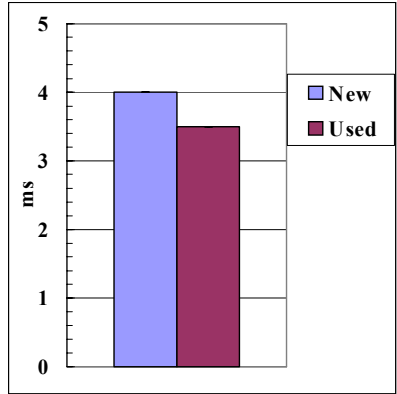

(c) Contact time $T_{C}$

Fig.16 Top spin applied by a professional tennis player for new and used gut strings showing average and standard errors.

Figure 17 shows an overview of the swing trajectory before and after impact in the case of a top spin shot performed by an amateur tennis player.

Figure 18 shows calculation results. In this case, it compares top spin shots by professional and amateur players using brand new gut strings. The professional shot shows little difference from that of its amateur counterpart in terms of post-impact ball velocity, whereas the spin rate is 1.6 times $(60 \%)$ greater. Furthermore, since the spin rate is higher, the contact time between the ball and gut is 1.3 times $(30 \%)$ longer. We assume that a substantial shock vibration is transmitted to the player's upper arm by a forceful top spin shot. However, a high spin rate reduces the shock force and shock vibration transmitted to the hand at impact, even for powerful shots. 


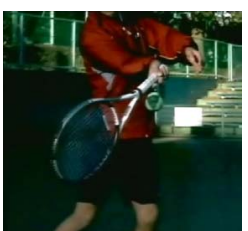

$0.0 \mathrm{~ms}$

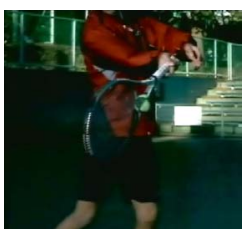

$7.5 \mathrm{~ms}$

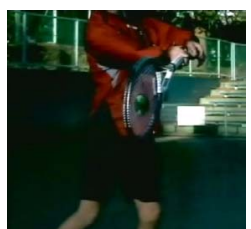

$15.0 \mathrm{~ms}$ (impact)

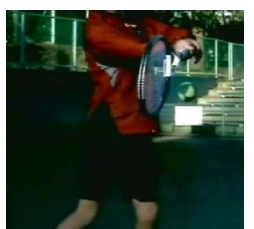

$22.5 \mathrm{~ms}$

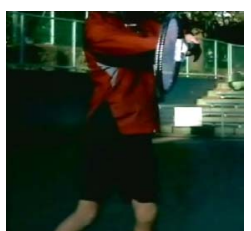

$30 \mathrm{~ms}$

Fig. 17 Top-spin swing applied by an amateur tennis player.

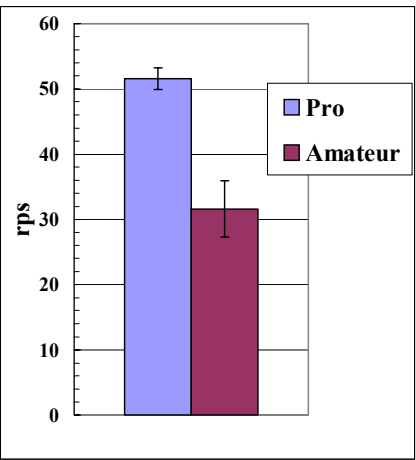

(a) Ball spin rate $\omega$

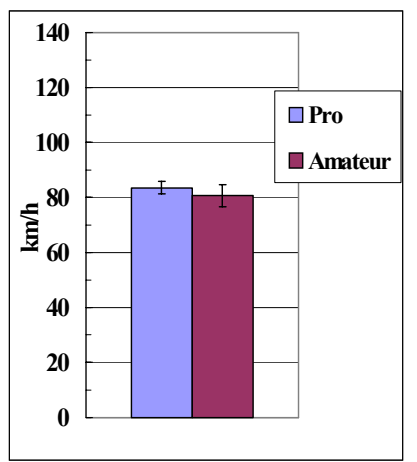

(b) Post-impact ball velocity $V_{B}$

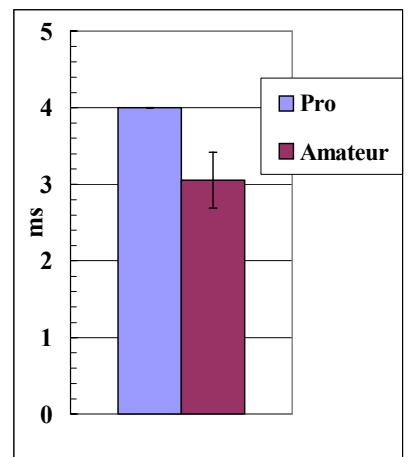

(c) Contact time $T_{C}$

Fig. 18 Comparison of top-spin performances of professional and amateur players using brand-new gut strings (showing average and standard error).

\section{Conclusion}

We have quantitatively investigated the effects of notches formed at intersections on top-spin performance by ultrahigh-speed video analysis for nylon and gut (natural string: cattle intestine) strings.

(1) A racket strung with nylon strings and used for $3 \mathrm{~h}$ a day for about 1 week has a $40 \%$ lower average spin rate and $8 \%$ shorter average contact time than brand-new strings. When a lubricant is applied to notched strings, the spin increases substantially, but the spin performance fails to reach that of brand new strings without notches.

(2) In the case of gut, with a notched racket that had been previously used by a professional tennis player, the spin rate decreased by an average of $70 \%$ and the contact time shortened by an average of $13 \%$, results which support the top-spin mechanism presented in the previous report ${ }^{(14)}$.

(3) There is little difference in the post-impact ball velocities of shots by professional and amateur tennis players. However, the spin rate and contact time achieved by the professional are an average of 1.6 and 1.3 times greater than their amateur counterparts, respectively. The long contact time reduces the shock force at impact and the shock vibration transmitted to the hand, even for powerful shots.

(4) As is becoming increasingly well known, experimental results suggest neither polymer strings nor gut lose much of their rebound ability as they get older ${ }^{(16)}$. However, their spin performances decrease dramatically.

Strings known as "spin gut" are commercially available. These include types which have angular, unusually shaped cross-sections in contrast to the round cross-section of ordinary gut, and types in which fine fibres are positioned around ordinary gut, forming convex shapes on the gut surface. This is an attempt to try to make it easier to apply spin by catching the ball on the angles or convex formations on the gut surface, increasing the friction, and thereby increasing the spin rate. Accordingly, research into the spin properties 
of strings has started to pursue this direction. However, since the main (vertical) and cross (horizontal) gut strings are woven so that they alternate behind and in front of one another when a racket is strung (by regulation), there are already sufficient protrusions on the gut surface. In contrast to the protrusions formed on spin gut which are less than 1/10 of the gauge diameter (diameter of the gut), the bumpiness caused by the main and cross strings is the same as the gauge diameter. It is thus clear which would be the dominant generator of frictional force when the ball is caught; this has been acknowledged by many general players.

The hard, low-friction polyester strings, on the other hand, are not only hard to break, they do not overshoot (i.e., the ball remains in the court even when hit powerfully) as has been found empirically by players; this agrees with the experimental results of the present report, that the more spin is applied, the greater the reduction in the post-impact ball velocity. The design concept of manufactures and the feelings of top players are contradictory, and there is a degree of confusion among general players. We wish to highlight the necessity for a qualitative change in the conventional design concept for spin gut. Even newly strung brand-new strings will develop notches at the intersections, leading to a reduction in spin performance.

We will discuss spin behavior for the case of underspin (slicing) and the effects of the fuzz on balls exerted on spin and ball control in another report.

\section{Acknowledgments}

The present report drew together, primarily, experimental data obtained during the course of the production of an NHK television program. We wish to express our deepest thanks to Mr. Hirotaka Maruyama of the Japan Broadcasting Corporation, Mr. Isao Matsuyama and Mr. Masatoshi Satoh of Documentary Japan Inc., the Japan Tennis Association, the professional tennis player Mr. Masatoshi Miyazaki, and to everyone else who cooperated with us.

\section{References}

(1) Kawazoe, Y., Effects of String Pre-tension on Impact Between Ball and Racket in Tennis, Theoretical and Applied Mechanics, Vol.43, 1994, pp.223-232.

(2) Kawazoe, Y., Tanahashi, R. and Casolo, F., Experimental and theoretical criticism of the effectiveness of looser strings for the reduction of tennis elbow. Tennis Science \& Technology 2, 2003, pp.61- 69. London: International Tennis Federation.

(3) Kawazoe, Y., Impact phenomena between racket and ball during tennis stroke, Theoretical and Applied Mechanics, Vol.41, 1992, pp.3-13.

(4) Kawazoe, Y., Coefficient of restitution between a ball and a tennis racket, Theoretical and Applied Mechanics, Vol.42, 1993, pp.197-208.

(5) Kawazoe, Y., Mechanism of High-Tech Tennis Rackets Performance, Theoretical and Applied Mechanics Japan, Vol.51, 2002, pp.177-187.

(6) Kawazoe, Y., Computer aided performance prediction and estimation system for a tennis racket in terms of power and stability. The engineering of Sport 5, Vol.2, 2004, pp.633-640.

(7) Kawazoe, Y., Experimental Identification of Hand-held Tennis Racket Characteristics and Prediction of Rebound Ball Velocity at Impact, Theoretical and Applied Mechanics, Vol.46, 1997, pp.165-176.

(8) Kawazoe, Y., Mechanism of Tennis Racket Performance in terms of Feel", Theoretical and Applied Mechanics, Vol.49, 2000, pp.11-19.

(9) Kawazoe, Y., Tomosue, R., Yoshinari, K. and Casolo, F., Prediction of the shock vibrations at the wrist joint with the new large ball compared to the conventional ball 
impacted to the tennis racket during forehand stroke. Tennis Science \& Technology 2, 2003, pp.105-112. London: International Tennis Federation.

(10) Kawazoe, Y., Casolo, F., Tomosue, R. and Yoshinari, K., Performance prediction and estimation system for tennis racket in terms of player's wrist joint shock vibrations. The engineering of Sport 5, Vol.1, 2004, pp.393-399.

(11) Lindsey, C., Did you Hear What the Ball Said to the Strings? - "BITE ME ! ", Racquet Tech (The Official Publication of the United States Racquet Stringers Association), October 2003, p.2.

(12) Cross, R., Oblique impact of a tennis ball on the strings of a tennis racket, Sport Engineering, Vol.6, 2003, pp.235-254.

(13) Goodwill, S \& Haake, S., Effect of string tension on the impact between a tennis ball and racket, The engineering of Sport 5 , Vol.2, 2004, pp.3-9.

(14) Kawazoe, Y., Okimoto, K. and Okimoto, K., Mechanism of Racket Spin Performance in Tennis: Spin Performance Improvement by Lubrication of String Intersections with Super High Speed Video Analysis, Trans. JSME, Vol.72, No.718, 2006, pp.1900-1907. (in Japanese)

(15) Goodwill, S \& Haake, S., Why were 'spaghetti string' rackets banned in the game of tennis?, The engineering of Sport 4, 2002, pp.231-237. Blackwell Science, Oxford.

(16) Cross, R., Properties of tennis equipment: balls that bite, rackets that don't vibrate and strings don't make any difference, Tennis Science \& Technology 2, 2003, pp.17-29. International Tennis Federation,

(17) Kawazoe, Y., Takeda, Y., Nakagawa, M., Casolo, F., Tomosue, R. and Yoshinari, K., Prediction of Impact Shock Vibrations at Tennis Player's Wrist Joint: Comparison between Conventional Weight Racket and Light Weight Racket with Super Large Head Size, Journal of System Design and Dynamics, Vol.4, No.2, 2010, pp.331-347.

(18) Kawazoe, Y., Takeda, Y. and Nakagawa, M., Performance Prediction of Active Piezo Fiber Rackets in Terms of Tennis Power, Journal of System Design and Dynamics, Vol.4, No.1, 2010, pp.77-90. 